\title{
Intrusion Detection with CUSUM for TCP-Based DDoS
}

\author{
Fang-Yie Leu and Wei-Jie Yang \\ Department of Computer Science and Information Engineering, \\ Tunghai University, Taiwan \\ leufy@thu.edu.tw
}

\begin{abstract}
DDoS(Distributed Denial of Service) is the most troublesome attack nowadays, especially for those people whose operational environment relies on network services and/or the Internet. However, attackers often penetrate innocent routers and hosts to make them unwittingly participate in such a large scale attack as zombies or reflectors. In this paper, we propose an Intrusion Detection System (IDS), named CUSUM Intrusion Detection System (CIDS), which invokes CUSUM as its detection algorithm and logically divides Internet into many autonomous network management units (NMUs), each deploys a CIDS to discover attacks and identify what role a client in such an attack acts as.
\end{abstract}

\section{Introduction}

As Internet grows quickly, its network security has recently attracted researchers' attention. The threats caused by intruders may be losing disclosing secrets or reducing market opportunity. Intrusion Detection Systems (IDSs) or firewalls are prosperously deployed to protect network systems. Generally, IDS based on detective features can be classified into Network-based, host-based and hybrid of them. However, most of them are behavior-based and most of traditional Network-based IDSs (NIDSs) detect abnormal network behavior by monitoring if network traffic exceeds its threshold or not. However, such systems are no longer appropriate and sufficient nowadays due to the divergence of Internet activities.

DoS (Denial of Service) and DDoS (Distributed Denial of Service) are notorious attacks owing to easy commencement and tremendous destruction. Some popular web sites, such as Yahoo, Amazon, and eBay, had ever been attacked by them in February 2000. These attacks can be simply issued by attacking tools which with friendly user interfaces are available on Internet. Therefore, attackers can easily produce huge and legitimate traffic of the same or different protocols to flood victims. Gibson [1] foretold that at 2:00 AM, January 11th 2002, grc.com would be blasted off by an advanced malicious packet flood. What surprising us was that this attack came from more than 200 non-spoofed core routers. This attack, called Distributed Reflective Denial of Service (DRDoS), as shown in [1] is an extension of the DDoS by deploying some clients as reflectors to launch attack packets. Moreover, SYN_ACK flooding packets responded from reflectors through HTTP (web) port 80 or some other frequently used ports are hard to distinguish from those of normal connections.

Also, most DDoS and DRDoS attacks are TCP based since TCP Three-way handshake lacks a verification mechanism. In this paper, we proposed a detecting 
system, named CUSUM Intrusion Detection System (CIDS), which invokes the Cumulative SUM (CUSUM) algorithm [2,3] to discover attacks and the role a node acts as within an attack. To develop CIDS, we first analyze TCP-based attacks and the drawback of TCP protocol. Next, abnormal network behaviors occurred at victim, reflector and attacker (zombie) sites during an attack are addressed. Finally the ways to detect DDoS and DRDoS attacks by invoking CUSUM algorithm are designed.

\section{Related Work}

\subsection{DDoS Attack}

DoS/DDoS after initiated will continue until it is terminated by hackers or mitigated by victims. Situation becomes worse when their control messages are encrypted to evade IDS's detection. Many countermeasures are proposed to defense against DDoS attack $[4,5]$. Some prevent clients from being zombie agents. Some try to relieve victims from attack damage. IP traceback mechanism [6-10] is also one of the major security aspects. Most solutions mainly focus on how to identify traffic sources. The main concerns of designing a detector are less computation and fast detection.

\subsection{TCP Three-Way Handshake and SYN Flood Attack}

Establishing a TCP session requires the TCP Three-Way Handshake, whereas normally disconnecting a TCP connection needs to exchange four packets [11]. After accepting a SYN request, server allocates resources for the request and replies SYN_ACK. This is so called half-open connection. A traditional SYN Flood delivers large amount of SYN packets with randomly spoofed source IPs to consume server's resources or network bandwidth. Unfortunately, only few of the spoofed addresses really alive and then send RST to terminate the non-existing connections. Most source addresses reply no SYN-ACK packets resulting in server reserving too many resources so that it is unable to provide services to other legal users.

\subsection{Distributed Reflective Denial of Service (DRDOS) Attacks}

Paxson [12] had deeply analyzed reflector attacks in early 2001. Any IP host that returns a reply as receiving a packet may act as the reflector. Using traceback techniques, we can trace the reflectors, but can not locate and identify who issues the attack.

\section{CUSUM Intrusion Detection System (CIDS)}

We first divide Internet into many autonomous Network Management Units (NMUs). An enterprise intranet and a campus network are examples. All ingress and egress packets of an NMU are detected by CIDS, an IDS integrating HIDS(Host-based IDS) and NIDS (Network-based IDS) properties to detect DDoS and DRDoS, as shown in Fig. 1, by means of collecting packets flowing through internal router as the observed event sequence. Like most detection systems, CIDS compares the observed sequence with users' normal behaviors recorded in profiles to find out the significant discrepancy and difference. A procedure, named Change-Point Detection designed to 
detect the change point of a network behavior, is as follows. First, compare observed event sequence with user profiles. If any difference is significant, identify the time point the change happens so as to real time discover when the attack starts. Second, CUSUM $[2,13]$ is deployed to sequentially monitor input random variables. A simple parametric approach $[13,14]$ is often too simple to accurately model a network session due to the complexity of Internet. CUSUM with the characteristics of sequential and nonparametric light computation load can make CIDS work online.

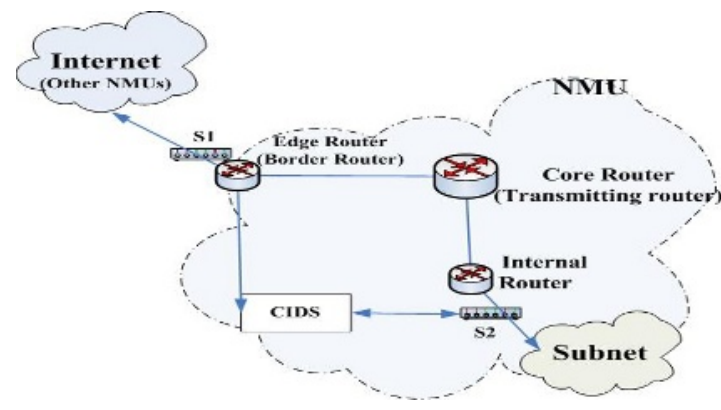

Fig. 1. Framework of a NMU (S1 and $\mathrm{S} 2$ are switches)

\subsection{CUSUM}

CUSUM can detect sharp but continuous increase. Some of its assumptions are as follows. First, let $X_{n}$ be the packets collected by CIDS in a sampling time $\Delta n$ and $\bar{X}$ the mean value of random sequence $X, X=\left\{X_{n}, n=0,1,2 \ldots\right\}$. Second, let $Z=\left\{Z_{n}, n=0,1,2 \ldots\right\}$ with a, where $Z_{n}=X_{n}-a$ and $a$ is the peak value of normal traffic for a specific network status so that all elements of $Z$ are negative, so is $\bar{Z}$. When a change, such as flow-based attack, occurs, $Z_{n}$ will suddenly increase to positive, as illustrated in Fig. 2. $Z_{k} \geq \bar{Z}+h$, for some $k$, indicates an attack possibly starts where $k$ is the smallest $n$ and $h$ the threshold of abnormal network traffic. $\Delta_{k}$ is then considered as the change point of network traffic. $y_{n-1}+Z_{n} \leq 0$ shows there is no attack. CUSUM accumulates $Z_{n}, m \geq k$, with formula(1) which is the recursive version of the non-parametric CUSUM algorithm[2].

$$
y_{n}=\left(y_{n-1}+Z_{n}\right)^{+}, y_{0}=0
$$

where $x^{+}=x$ if $x>0$ and 0 otherwise. $Z_{n}, n>k$, may now be positive or negative.

The decision function at $\Delta p$, say $d_{p}\left(y_{p}\right)$, is as follows:

$$
d_{p}\left(y_{p}\right)=\left\{\begin{array}{ll}
1 & \text { if } y_{p}>N \\
0 & \text { else }
\end{array} .\right.
$$

where $N$ is the threshold of an attack. ' 1 ' indicates an attack occurs, while ' 0 ' shows network operates normally. 


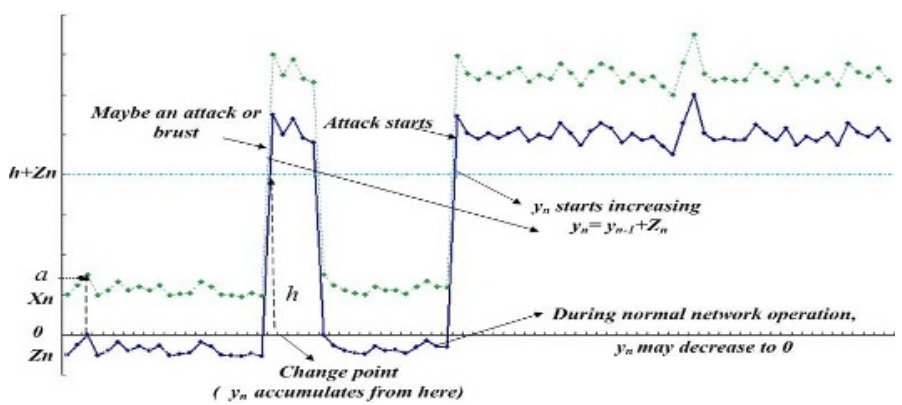

Fig. 2. An example of CUSUM

\subsection{TCP SYN Flood}

In the following, we traditional and reflective TCP Flood attacks in detail. A client inside an NMU may act as a victim, reflector, or an attacker (zombie).

During an attack, a zombie sends a large amount of SYN packets of random source IPs to victim as shown in Fig. 3(a). Due to SYN-ACK packets never replying back, $|O-S Y N|$ and $\left|I-S Y N_{-} A C K\right|$ at zombie significantly differ where $|X|$ represents the number of $X, O-S Y N$ and $I-S Y N_{-} A C K$ stand for outgoing SYN and incoming SYN_ACK respectively. However, $|I-S Y N|$ and $\left|O-S Y N_{-} A C K\right|$ at victim are similar and huge.

Wang et al. [11] mentioned, before a normal TCP session ended, a SYN packet would result in a returned FIN packet, so does SYN-ACK. Generally, a RST packet following a TCP packet, e.g., SYN, SYN_ACK, URG or PSH, represents one of the three cases: (1) terminating a TCP session; (2) aborting a TCP request; (3) destinating a packet to a closed port or an unconnected node.

(a) T C P SY N Flood

(b) Reflective T CP SY N Flood - victim replies RSTs

(c) Reflective TCP Flood - Victim replies nothing
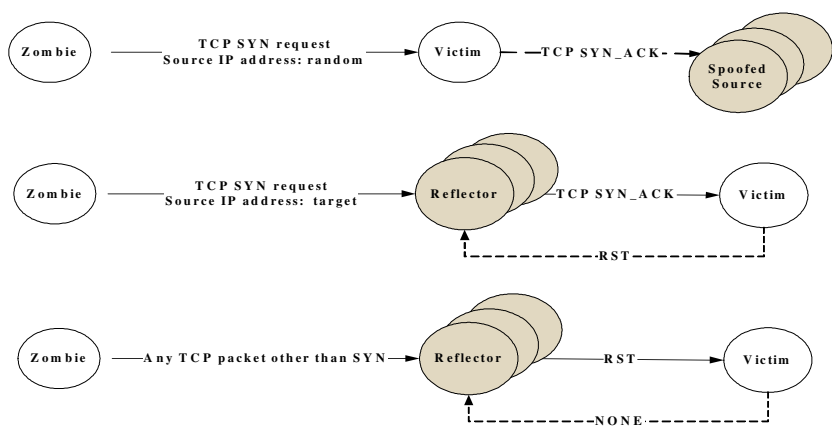

Fig. 3. Attack nodes of TCP Flooding

\section{(1) Traditional SYN Flood}

Normally, ISYN|+|SYN/ACK| almost equals to |RST $|+|$ FIN $\mid$ at a node. The ratio of active RST packets, generated by the first two cases which are strongly related to 
SYN, is about $75 \%$ of all RST packets, thus taking 3/4 as our experienced cumulated normal amount. The remaining $1 / 4$ resulted from the third is treated as background noise to improve detection sensitivity and decrease false alarms [11]. CIDS considers SYN-FIN, SYN_ACK-FIN, and SYN-RST ${ }_{\text {active }}$ pairs normal behaviors at victim. Those deviated from normal indicate the node is now under a TCP SYN Flood attack.

\section{(2) Reflective TCP Flood}

During a reflective attack, zombies send some level of volume of TCP SYN requests to each reflector [1]. Two facts are useful in detecting such an attack. First, as receiving a spoofed request, a reflector replies a SYN_ACK to victim which, as shown in Fig. 3(b), will send a RST back. Second, when any TCP packet other than SYN, such as URG, PSH, FIN or a TCP packet without a flag, arrives without any previous handshake, reflector replies a RST to victim which answers nothing, as illustrated in Fig. 3(c). An abnormal increase of $|O-R S T|$ at victim implies the existence of reflector which may be in underlying or other NMU.

Wang et al. [3, 11] proposed the difference between $\left|I-S Y N_{-} A C K\right|$ and $|O-S Y N|$ is helpful in discovering the existence of attacker in situation of both DDoS and DRDoS. From the second facts stated above, we discover that a $\left|I-S Y N_{-} A C K\right|$ and $|O-R S T|$ packets at a victim hugely increase, so do $\left|O-S Y N_{-} A C K\right|$ and $|I-R S T|$ at a reflector. But $|O-S Y N|$ and traffic generated by other TCP packets at both nodes do not. The phenomenon is extremely useful in detecting reflector and victim.

\subsection{Detecting TCP SYN Flood}

CIDS monitors network behavior in order to collect corresponding $X_{n}$.

\section{(1) Victim}

Let $\left|S_{S Y N}\right|,\left|S_{F I N}\right|,\left|S_{S Y N_{-} A C K}\right|$ and $\left|S_{R S T}\right|$ respectively represent numbers of SYN, FIN, SYN_ACK and RST packets observed within a sampling interval $\Delta n$, but ignoring packet direction. Let $X_{V n}$ be the normalized $X_{n}$, obtained by formula (3), so that $X_{V n}$ is independent of network size and ordinary traffic since normally the ratio between request pairs(SYN-SYN_ACK) and reply pairs(RST-FIN) is relatively stable.

$$
X_{V n}=\frac{\left(\left|S_{S Y N}\right|+\left|S_{S Y N_{-} A C K}\right|\right)-\left(\left|S_{F I N}\right|+\left|S_{R S T}\right|\right)}{\left(\left|S_{F I N}\right|+\left|S_{R S T}\right|\right)}
$$

With $X_{V n}$, a victim can be discovered. However, [11] mentioned that most TCP connections lasted 12-19 seconds. In this paper, $\Delta n=10 \mathrm{sec}$, therefore, FIN and RST packets and their corresponding SYN and SYN_ACK packets always appear in different intervals. Let the sampling time correction (delay) of $\left|S_{F I N}\right|$ and $\left|S_{R S T}\right|$ be $\beta$, i.e., $\Delta n_{(F I N, R S T)}=\Delta n_{\left(S Y N, S Y N_{-} A C K\right)}+\beta$, where $\Delta n_{(F I N, R S T)}$ and $\Delta n_{\left(S Y N, S Y N_{-} A C K\right)}$ are the sampling time of $S_{F I N}\left(S_{R S T}\right)$ and $S_{S Y N}\left(S_{S Y N_{-} A C K}\right)$ respectively. According to [11], $\beta=10$ can balance the sensitivity and detection accuracy. 
Generally, few RST packets are generated under normal operation. The major reasons that $\left(\left|S_{S Y N}\right|+\left|S_{S Y N_{-} A C K}\right|\right)$ and $\left(\left|S_{F I N}\right|+\left|S_{R S T}\right|\right)$ significantly defer are as follows.

(1) The long-lived TCP connection may result in incomplete SYN-FIN pairs.

(2) The retransmission of TCP requests (SYN) may also conduct the difference due to connecting to a non-existing or failed node.

(3) An RST is generated long after its corresponding SYN_ACK.

Therefore, by monitoring $X_{V n}$, when the parameters $h, a$ and $N$ of the given $X_{n}$, say $h_{v}, a_{V n}$ and $N_{V}$, are defined where $Z_{v n}=X_{v n}-a_{v n}, h_{v}=2$ and $a_{V n}=1$ [3], decision function can then determine if a node $\mathrm{V}$ is a victim of a traditional TCP SYN Flood attack or not. Let

$$
X_{V n}{ }^{\prime}=\frac{\left|I-S Y N_{-} A C K\right|-|O-S Y N|}{|O-S Y N|}
$$

Similar to $X_{V n}$, given $a_{V n}{ }^{\prime}, h_{v}{ }^{\prime}$ and $N_{V}{ }^{\prime}$, CIDT can decide a node $V$ is now a victim of a reflective TCP SYN Flood or not. This can be very accurate since intruder can not decrease the value of $X_{V n}$ ' by generating spoofed O-SYN for $V$.

For detecting victim in Fig. 3(c), let

$$
X_{V n} "=\frac{|I-R S T|-a v g|I-R S T|}{a v g|I-R S T|}
$$

where $a v g$ represents average.

\section{(2) Attacker (Zombie)}

At the attacker side, let

$$
X_{Z n}=\frac{|O-S Y N|-\left|I-S Y N_{-} A C K\right|}{\left|I-S Y N_{-} A C K\right|}
$$

Given $h_{Z n}, a_{Z n}$ and $N_{Z n}$, CUSUM can determine if a node is zombie or not for both of traditional and reflective TCP SYN Flood attacks.

\section{(3) Reflector}

Let

$$
X_{R n}=\frac{|I-R S T|-a v g|I-R S T|}{\operatorname{avg|I-RST|}}
$$

Given $h_{R}, a_{R}$ and $N_{R}$, a reflector of a reflective TCP SYN Flood can be detected. However, observing $X_{R n}$ may be insufficient since its $|I-R S T|$ may be not huge enough, especially when many reflectors are deployed. An abnormal increase of both $X_{R n}$ and $X_{Z n}$, in the same or different NMUs, indicate that reflectors exist. This finding can be the basis for CIDS to discover who the reflectors are. However, as reflector and zombie are located in different NMUs, they have to communicate with each other [15]. 
Besides, to detect reflector with situation in Fig. 3 (c), let

$$
X_{R n}{ }^{\prime}=\frac{|O-R S T|-a v g|O-R S T|}{\operatorname{avg}|O-R S T|}
$$

Given $h_{R n}{ }^{\prime}, a_{R n}{ }^{\prime}$, and $N_{R}{ }^{\prime}$, reflector can be then detected. Combining $X_{R n}$ with $X_{R n}{ }^{\prime}$, CIDS can detect a reflector by monitoring $\left|\mathrm{S}_{R S T}\right|$.

Besides, supporting factor to discriminate the reflector of Fig. 3(b) and victim of Fig. 3(c) is required. Let

$$
X_{D}=\frac{\left|S_{R S T}\right|+\left|S_{F I N}\right|}{\left|S_{S Y N}\right|+\left|S_{S Y N}\right|}
$$

$X_{D}$ is a stable under normal network operation. During a reflective TCP SYN Flood as the one in Fig. 3(b), $\left|S_{S Y N}\right|$, $\left|S_{S Y N_{-} A C K}\right|$ and $\left|S_{R S T}\right|$ increase, but $\left|S_{F I N}\right|$ does not, and $|I-R S T|$ is almost equal to $\left|O-S Y N_{-} A C K\right|$. Thus $X_{D}$ does not increase. But in Fig. 3(c), only $|I-R S T|$ becomes large resulting in abnormal increase of $X_{D}$.

\section{Defending with Autonomous NMU}

CIDS is originally deployed as the detection component of Intrusion Forecast and Traceback System (IFTS) [15] which integrates intrusion detection and traceback and provides some policies against DDoS attack. An NMU deploys an IFTS as it security system of which a hash-based Traceback mechanism[6] is developed so that tracing intruders can be performed once one attack packet has been detected.

Besides, an IFTS has an Intrusion Response Manager (IRM), the communication center of an NMU, offering the Certification Authority for exchanging information among NMUs. The information consists of tracing messages and a request for filtering attack packets. Thus, NMUs can defeat DDosS through collaborative cooperation with others, especially by deploying CIDS as its powerful DDoS/ DRDoS detector.

Detecting malicious behavior, CIDS analyzes abnormal immediately. Real attackers can be found through the MAC Address rather than spoofed IP addresses since we gather packet information before it flows through the first router of an NMU [15].

Once a suspected attacker, reflector or a victim is discovered, CIDS asks IRM to notify the victim's NMU, may be local or remote, which, after confirming some packets coming from the attacker, in turn asks its traceback mechanism to trace to the real zombies and to filter out attack packets in order to mitigate damage.

\section{Experiments}

Our experimental environment is as follows. The observed interval $\Delta n_{(F I N, R S T)}=\Delta n_{(F I N, R S T)}=\beta=10 \mathrm{sec}$. Also, normally the $|O-S Y N|$ at a node is always larger than $\left|I-S Y N_{-} A C K\right|$ due to the SYN loss and subsequent retransmission of a 
TCP SYN request. Although Wang et al. $[3,11]$ treated them as white noise. But they cause negative $\overline{X_{V n}}$ 'under normal network operation, as shown in Fig. 4. Therefore, let $Z_{V n}{ }^{\prime}=X_{V n}{ }^{\prime}$, i.e., $\mathrm{a}=0$.

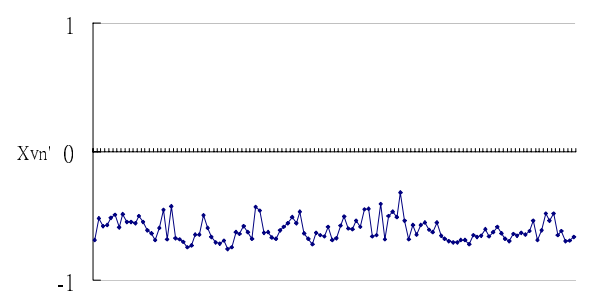

time

Fig. 4. $X_{V n}$ ' with negative $\overline{X_{V n}}$ ' (Reflective TCP SYN Flood)

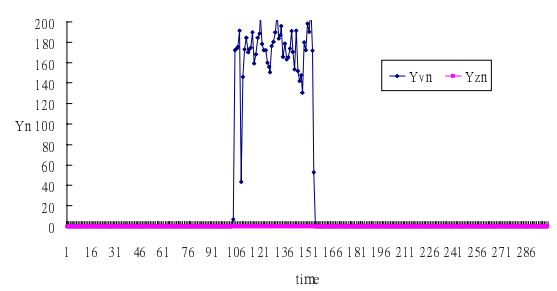

Fig. 6. $y_{V n}$ under DDoS attack

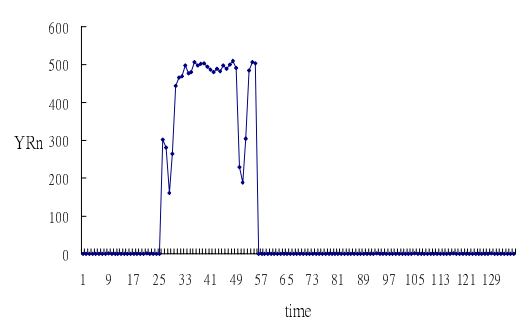

Fig. 8. $y_{A n}$ during an DRDoS

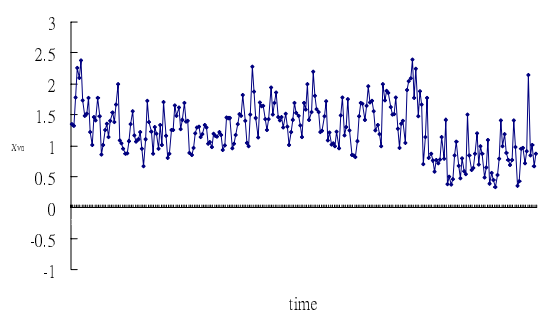

Fig. 5. Example of $X_{V n}$ (TCP SYN Flood)

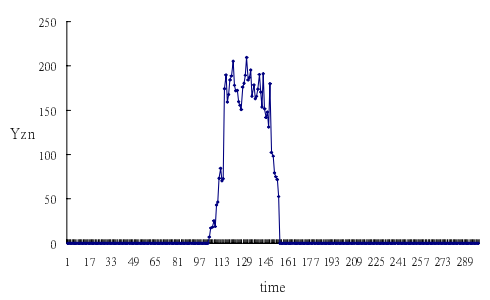

Fig. 7. $y_{Z n}$ under DDoS

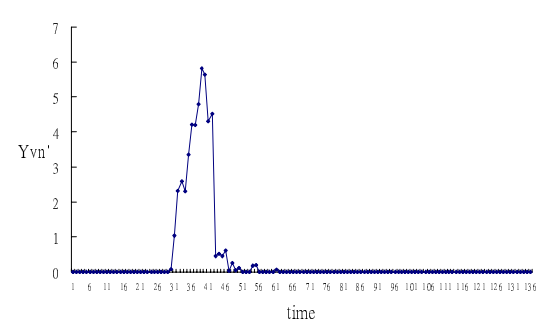

Fig. 9. $\mathrm{y}_{\mathrm{Vn}_{\mathrm{n}}}$ ' under DRDoS

Due to loss of SYN packets, we give the customized $h_{z}=4, a_{Z n}=2.5$ and $N_{z}=4$ for $X_{V n}$ according to our offline observation shown in Fig. 5. The system with the properties stated above is involved as our testbed.

The first experiment is a DDoS attack. Victim and zombie are located in different NMUs, say $\mathrm{NMU}_{\mathrm{V}}$ and $\mathrm{NMU}_{\mathrm{z}}$ respectively. We can see that $\mathrm{y}_{\mathrm{vn}}$ shown in Fig. 6 
increases to huge immediately, but $y_{Z n}$ of each node in $\mathrm{NMU}_{\mathrm{v}}$ does not, showing that the attack comes from other NMU. This occurrence can identify a victim from local nodes. Within $\mathrm{NMU}_{\mathrm{Z}}$, some node's $y_{Z n}$ increases to exceed $N_{z}$, as illustrated in Fig. 7, indicating this node is an attacker.

The second experiment is a DRDoS attack. Some $X_{R n}$ ' in $N M U_{R}$ increases and $y_{R n}$ soon exceeds $N_{R}$, as shown in Fig. 8, indicating it is a reflector. Fig. 9 illustrates that $y_{V n}{ }^{\prime}$ in $N M U_{V}$ also exceeds its threshold as an attack happens, stating that victim is now under attack.

\section{Conclusion}

With customized CUSUM parameters, all the situations in NMU can be totally monitored by CIDS with the decision function $d_{n}\left(y_{n}\right)$ to quickly detect the role a host may act as in real time. The attack can be defeated in the initial stage since the zombie can be located by its local CIDS.

Besides, detecting reflector can help original IFTS traceback mechanism to overcome tracing limitation. Attacking sources in a DRDoS attack can be then traced after reflectors are identified, whereas logical attacks can be traced directly.

With the assist of lightweight detecting approach, like CUSUM, and the help of traceback and filtering mechanisms, we can mitigate the victim from the progressing attack. CIDS can offer us more defending power against the distributed malicious behavior so as to carry out a more secure Internet.

\section{References}

1. Gibson, S.: DRDoS, Distributed Reflection Denial of Service. http://grc.com/dos/drdos.htm.

2. Brodsky, B.E., Darkhovsky, B.S.: Nonparametric Methods in Change-point Problems. Kluwer Academic Publishers (1993).

3. Wang, H., Zhang D., Shin, K.G.: Change-Point Monitoring for the Detection of DoS Attacks. IEEE Transactions on Dependable and Secure Computing, vol. 1, (Oct.-Dec. 2004) 193- 208.

4. Chang, R.K.C.: Defending against flooding-based distributed denial-of-service attacks: a tutorial. IEEE Communications Magazine, vol. 40 ( Oct. 2002) 42-51.

5. Leu, F.Y., Hung J.J., Hung, C.H.: UDAIDTS : Union Defense with Active Intrusion Detection and Hash-based Traceback System. Proceedings of International Conference on Information Management Conference, Taiwan (July 2003) (in Chinese).

6. Snoeren, A.C. et al.: Single-Packet IP Traceback. IEEE/ACM Transaction on Network, vol. 10, no. 6 (Dec. 2002) 721-734.

7. Belenky, A., Ansar, N.: On IP traceback. IEEE Communications Magazine, vol. 41 (July 2003) 142-153.

8. Dawn Song, X.D., Perrig, A.: Advanced and Authenticated Marking Schemes for IP Traceback. Proceedings of 20th Annual Joint Conference of the IEEE Computer and Communications Societies, vol. 2 (April 2001) 878-886. 
9. Bellovin, S.M.: ICMP Traceback Messages. IETF draft, 2000. http://www.research.att.com/smb/papers/draftbellovin -itrace-00.txt, (refer to in March 2005).

10. Savage, S. et al.: Network Support for IP Traceback. IEEE/ACM Transaction on Network, vol. 9, no. 3 (June 2001) 226-237.

11. Wang, H., Zhang, D., Shin, K.G.: Detecting SYN Flooding Attacks. Proceedings of IEEE Computer Communications (June 2002) 1530-1539.

12. Paxson, V.: An Analysis of Using Reflectors for Distributed Denial-of Service Attacks. Computer Communication Review, 31(3) (July 2001) 38-47.

13. Mirkovic, J., Prier, G., Reiher, P.: Attacking DDoS at the Source. Proceedings of IEEE International Conference on Network Protocols (Nov. 2002) 312-321.

14. Gil, T.M., Poletter, M.: MULTOPS: A Data-Structure for Bandwidth Attack Detection. Proceedings of USENIX Security Symposium (Aug. 2001).

15. Leu, F.Y., Yang, W.J., Chang, W.K.: IFTS: Intrusion Forecast and Traceback based on Union Defense Environment. Proceedings of the 11th International Conference on Parallel and Distributed Systems (July 2005). 